\title{
Nephrotoxicity of once-daily dosing Gentamicin in Children
}

Hanako Funakoshi' ${ }^{1}$ Kahoru Fukuoka ${ }^{1}$, Yuta Aizawa ${ }^{1}$, Junichi Suwa ${ }^{2}$, Yuho Horikoshi ${ }^{1}$

1:Division of Infectious Diseases, Department of Pediatrics, 2:Department of Pharmacy, Tokyo Metropolitan Children's Medical Center, Tokyo, Japan.

Hanako Funakoshi, MD

Email:ha.co2ro@gmail.com

Background/Objectives

$\checkmark$ The aminoglycosides once-daily dosing (ODD) was increasingly reported in favor of efficacy and safety among adults and neonates compared to traditional three-times daily dosing. Experiences in aminoglycosides ODD are still limited in children outside of neonates.

$\checkmark$ Our aim of the survey was to evaluate nephrotoxicity in children who were treated with gentamicin ODD.

Methods

\section{- Design: A single-center, retrospective cohort}

- Site: Tokyo Metropolitan Children's Medical Center, Tokyo, Japan.

- Period: April, 2010 - March, 2017

- Inclusion criteria: Patients who were hospitalized at our hospital and treated with gentamicin ODD.

- Primary outcome: Nephrotoxicity during and after treatment with gentamicin

- Nephrotoxicity was evaluated according to the staging 1-3 of KDIGO guideline for acute kidney injury. (Table 3)

\section{Results}

A total of $\mathbf{2 6 8}$ cases with gentamicin ODD were observed. Trough level exceeded safe range of $1 \mu \mathrm{g} / \mathrm{mL}$ was

Table 1: Demographic characteristics of patients at enrolment

$\mathrm{N}=268$

Age, mont

21 [5-100]

Male $142(53.0)$

Reason for gentamicin

Urinary tract infection

$149(55.6)$

Bacteremia, Sepsis

$31(11.6)$

Cholangitis

$29(10.8)$

Prophylaxis in surgery of biliary atresia

$16(6.0)$

Administration of gentamicin

Duration, days

Trough initially tested, $\mu \mathrm{g} / \mathrm{mL}$

$5[3-7]$

$0.53[0.33-0.76]$

Data are presented as $\mathrm{n}(\%)$ or median [interquartile range]

$\mathrm{ODD}=$ Once-daily dose, $\mathrm{AKI}=$ Acute kidney injury,

UTI=Urinary Tract Infection, ICU=Intensive care unit,

$\mathrm{GP}=$ General pediatrics

observed in $\mathbf{2 5}$ cases (9.3\%). Among them,

no patients remained the acute kidney injury.

Table 2: Patients whose initial trough was over safety range

$\mathrm{N}=25$

\begin{tabular}{|l|r|}
\hline Age, month & $8[3-53]$ \\
\hline
\end{tabular}

\begin{tabular}{|l|l}
\hline Male & $12(48.0)$ \\
\hline
\end{tabular}

Underlying diagnosis $12(48.0)$

\begin{tabular}{l|l}
\hline Cardiac 14* & 14
\end{tabular}

\begin{tabular}{l|l}
\hline Neurological & 6 (4 individuals)
\end{tabular}

Gastrointestinal 5*

Reason for gentamicin

Urinary tract infection

Peritonitis, lleus

Administration of gentamicin

Duration, days

Trough initially tested, $\mu \mathrm{g} / \mathrm{mL}$

*One patient is overlapped.

Discussion/Conclusions

Gentamicin ODD was safe in children regarding nephrotoxicity.

Only mild and transient nephrotoxicity was observed in minority of patients at equivalent rate of traditional three-times daily dosing.

ODD has advantages in theoretical efficacy and safety therefore ODD may be an option for children.

\section{References:}

1) Best EJ et al. Once-daily gentamicin in infants and children: a prospective cohort study evaluating safety and the role of

therapeutic drug monitoring in minimizing toxicity. Pediatr Infect Dis J. 2011;30(10):827-832.

2) Kellum JA et al. KDIGO Clinical Practice Guideline for Acute Kidney Injury. Kidney International Supplements Vol 2.2012.
A total of 12 cases with Stage 1 and 2 AKI during or after treatment were observed. Table 3: AKI definition

\begin{tabular}{|c|l|l|}
\hline & \multicolumn{2}{|c|}{ Serum creatinine } \\
\hline Stage & Increase compared to baseline (within the prior 7 days) & Increase within 48 hours \\
\hline 1 & $1.5 \sim 1.9$ times & $\geqq 0.3 \mathrm{mg} / \mathrm{dL}$ \\
\hline 2 & $2.0 \sim 2.9$ times & \\
\hline 3 & 3.0 times or initiation of renal replacement therapy & $\geqq 4.0 \mathrm{mg} / \mathrm{dL}$ \\
\hline
\end{tabular}

Table 4: Patients with gentamicin-related nephrotoxicity

\begin{tabular}{|c|c|c|c|c|c|c|c|}
\hline \multirow[b]{2}{*}{ Ward } & \multirow{2}{*}{$\begin{array}{l}\text { Age, } \\
\text { month }\end{array}$} & \multirow{2}{*}{$\begin{array}{l}\text { Underlying } \\
\text { diagnosis }\end{array}$} & \multirow[b]{2}{*}{$\begin{array}{l}\text { Reason for } \\
\text { gentamicin }\end{array}$} & \multirow{2}{*}{$\begin{array}{l}\text { Initial } \\
\text { trough, } \\
\mu \mathrm{g} / \mathrm{mL}\end{array}$} & \multirow{2}{*}{$\begin{array}{c}\text { Duration of } \\
\text { gentamicin, } \\
\text { days }\end{array}$} & \multicolumn{2}{|c|}{ Nephrotoxicity, Stage } \\
\hline & & & & & & $\begin{array}{l}\text { During } \\
\text { treatment }\end{array}$ & $\begin{array}{c}\text { After } \\
\text { treatment }\end{array}$ \\
\hline \multirow{9}{*}{$\bar{\complement}$} & 1 & Cardiac & UTI & 1.02 & 5 & 1, Day7 & 0 \\
\hline & 51 & Cardiac & UTI & 0.31 & 3 & 1, Day 4 & 0 \\
\hline & 11 & Cardiac & UTI & 2.58 & 1 & 0 & $2,+7$ days \\
\hline & 1 & Urological & UTI & 0.51 & 4 & 1, Day8 & 0 \\
\hline & 3 & Cardiac & Bacteremia & 1.31 & 16 & 0 & $2,+7$ days \\
\hline & 3 & Cardiac & Bacteremia & 0.45 & 40 & 1, Day7 & 0 \\
\hline & 4 & Cardiac & Peritonitis & 0.91 & 7 & 1, Day5 & 1, +7days \\
\hline & 1 & Malrotation & Peritonitis & 1.08 & 3 & 1, Day3 & 1, +7days \\
\hline & 1 & Biliary & Cholangitis & 0.72 & 7 & 1, Day3 & 1, +7days \\
\hline \multirow{3}{*}{ ด } & 143 & Hematological & bacteremia & 0.27 & 15 & 1, Day7 & 0 \\
\hline & 3 & Renal & bacteremia & 0.24 & 13 & 2, Day 5 & 0 \\
\hline & 166 & Biliary & Cholangitis & 0.53 & 14 & 1, Day 11 & 0 \\
\hline
\end{tabular}

\section{Limitations:}

- We did not examine the efficacy.

- We evaluated nephrotoxicity using serum creatinine only.

- We did not investigate other drugs using concomitantly.

- The patients without renal impairment before using gentamicin might be selected. 